\title{
Accelerated Aging in HIV Patients
}

\author{
Keren Meir-Shafrir ${ }^{1}$ and Shimon Pollack ${ }^{1,2}{ }^{*}$ \\ ${ }^{\prime}$ Institute for Allergy, Immunology $\Theta^{2}$ AIDS, Rambam Health Care Campus, Haifa, Israel, and the ${ }^{2}$ Ruth \\ and Bruce Rappaport Faculty of Medicine, Technion-Israel Institute of Technology, Haifa, Israel
}

\begin{abstract}
Life expectancy has been increasing in the last few decades in the Western world and is accompanied by higher occurrence of age-related diseases like metabolic, cardiovascular, and renal diseases and also with a decline in immune functions. In HIV-infected people, due to the use of combination antiretroviral therapy (cART), life expectancy has increased. As a result, non-AIDS conditions which are age-associated have become more prevalent and appear earlier, resulting in accelerated aging in HIV patients. These non-AIDS conditions in HIV patients are associated with CD4 + T cell counts: lower counts are associated with higher rates of liver, cardiovascular, renal, and neurocognitive disorders. The effect of viral load and cART on the earlier occurrence of age-associated diseases is less significant than the CD4 count effect. Thus, the loss of immune functions in HIV-infected patients may enhance aging.
\end{abstract}

KEY WORDS: Aging, AIDS, HIV, immune senescence

HIV/AIDS came to the world's awareness over 30 years ago, with the first reports of young homosexual men, considered to be previously healthy, suffering from various types of opportunistic infections and profound cellular immunodeficiency. ${ }^{1}$ In the relatively short time since then, it has grown in scale to become a worldwide epidemic, with an estimated number of 34 million people living with HIV by 2011. ${ }^{2}$ The introduction of antiretroviral therapy (ART) in the middle of the 1990s has improved survival with HIV dramatically (Figure 1) and turned HIV infection, in effect, into a chronic condition. The percentage of people living with HIV/AIDS in USA who are over 50 years old is on the rise (Figure 2), and it is estimated that, by 2015 , people over 50 will constitute the majority of all people living with HIV/AIDS in USA. 5

\footnotetext{
Abbreviations: ART, antiretroviral therapy; BMD, bone mineral density; BMI, body mass index; cART, combination antiretroviral treatment; FRAM, Fat Redistribution and Metabolic Change in HIV Infection; HAD, HIV-associated dementia; HAND, HIV-associated neurocognitive disorder; HIVAN, HIV-associated nephropathy; HIVICK, HIV immune complex kidney disease; NRTIs, nucleoside reverse transcriptase inhibitors; SMART, Strategies for Management of Antiretroviral Therapy.
}

Citation: Meir-Shafrir, K., Pollack S. Accelerated Aging in HIV Patients. RMMJ 2012;3 (4):eoo25. doi:10.5041/RMMJ.10089

Copyright: (C) 2012 Meir-Shafrir and Pollack. This is an open-access article. All its content, except where otherwise noted, is distributed under the terms of the Creative Commons Attribution License (http://creativecommons.org/licenses/by/3.o), which permits unrestricted use, distribution, and reproduction in any medium, provided the original work is properly cited.

Conflict of interest: No potential conflict of interest relevant to this article was reported.

* To whom correspondence should be addressed. E-mail: Pollack@rambam.health.gov.il 


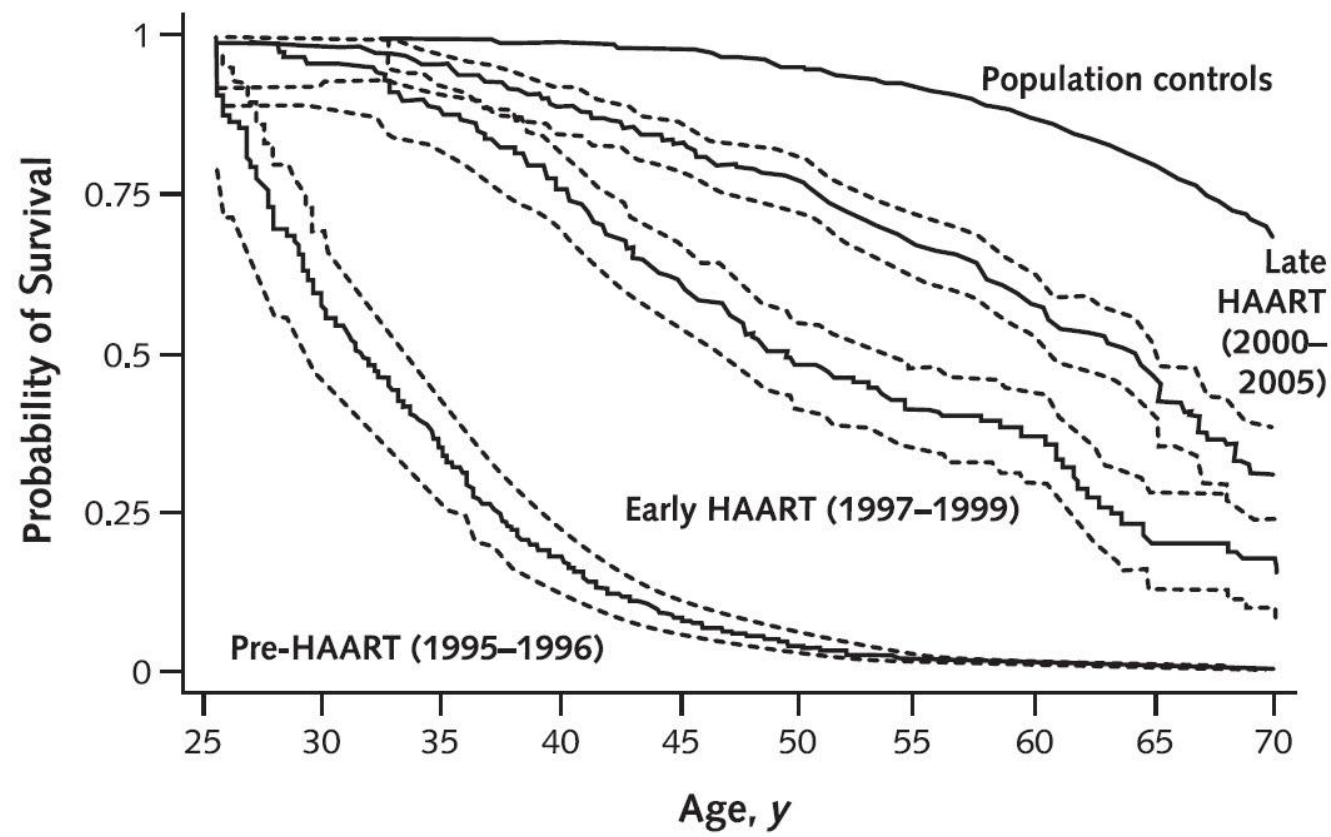

Figure 1. Estimated survival of 25-year-old HIV-infected and non-infected men in Denmark, 1995-2005. Persons with HIV infection are divided into 3 calendar periods of observation. Dashed lines indicate $95 \% \mathrm{Cls}$. HIV = human immunodeficiency virus; HAART = highly active antiretroviral therapy. Reprinted from Lohse et al. ${ }^{3}$ with permission of the American College of Physicians.

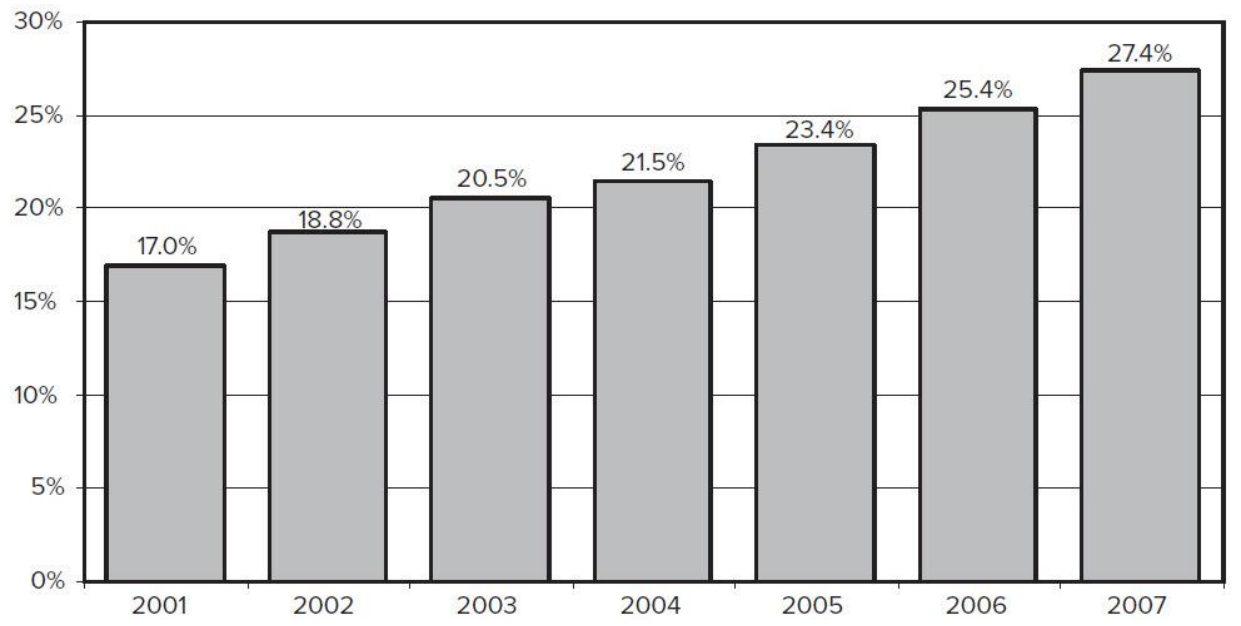

Figure 2. Estimated percentage of persons living with HIV/AIDS in USA who are older than 50, by year, 20012007.

Modified from Growing Older with the Epidemic: HIV and Aging, ${ }^{4}$ with permission.

As a result, we are encountering more chronic diseases typical of aging: cardiovascular disease, diabetes mellitus, dyslipidemia, osteoporosis and bone fractures, malignancies, and neurocognitive impairment. ${ }^{6}$ In addition, the accelerated aging of the immune system of HIV carriers has been demonstrated, 7 and this is accompanied by the parallel process of increased incidence of chronic diseases typical of aging and early signs of physical and functional frailty in this population. ${ }^{8}$ 
Accelerated aging may be a result of several factors, including HIV infection itself, ART sideeffects, and the aging of the immune system. It is now clear that the function of the immune system declines with age, but is the decline affecting the accelerated aging in HIV patients?

These evolving processes which interact with each other are becoming a major factor in treatment decisions of HIV carriers and shape research and clinical priorities, and they will be discussed further in this review.

\section{IMMUNOSENESCENCE AND HIV INFECTION}

Physiological aging of the immune system, termed immune senescence, is associated with a dysfunction in innate and adaptive immunity which diminishes the ability to respond to novel foreign antigens-vaccinations and infections. Similar changes in immune functions occur in people with chronic HIV infection but at a much younger age.

Changes seen in adaptive immune system manifest as lower naïve:memory $\mathrm{CD}_{4}$ ratio and enrichment of CD28-/CD57+/CD8+ effector T cells. 9 The latter are senescent cells with shorter telomeres and limited proliferative capacity. In addition, there are putative qualitative and quantitative changes in $\mathrm{T}$ regulatory cells ${ }^{10}$ and a decrease in the diversity of naïve $\mathrm{B}$ cells and a qualitative $\mathrm{B}$ cell dysfunction. ${ }^{11}$

In HIV carriers, peripheral blood lymphocytes show a tendency towards $\mathrm{T}$ cell senescence with enrichment of CD28-/CD57+/CD8+ T cells and inverted ratio of naïve/memory T cells, ${ }^{9}$ as seen in normal aging. However, the immunophenotypic changes seen in HIV-infected patients, though similar to the changes seen in HIV-negative individuals, appear $20-30$ years earlier..$^{12}$ Considering the innate immune system, peripheral blood monocytes from young HIV-positive individuals exhibit changes in phenotype, function, and telomere length that closely resemble those observed in elderly controls aged approximately 30 years older. Furthermore, these immune defects are not fully restored by ART. ${ }^{13}$ Another example of the same accelerated aging process is the telomere length of $\mathrm{CD}_{4}+$ and CD8+ T cells in HIV carriers, which resembles the telomere length of HIVnegative patients 38 years older. ${ }^{14}$
Chronic activation of the immune system probably contributes to the accelerated aging in HIV-infected patients. ${ }^{15}$ The chronic inflammatory process caused by the persistent immune activation is associated with the increased release of proinflammatory cytokines such as IL-6, IL-1 $\beta$, and TNF- $\alpha$ as well as pro-coagulants such as cystatin-C and D-dimer. ${ }^{16}$ These plasma biomarkers of inflammation decline dramatically with combination antiretroviral therapy (cART) administration, but do not normalize entirely. ${ }^{17}$ Chronic inflammatory manifestations are also seen in physiological aging and have been implicated in the development of cardiovascular disease in aged people. Chronic inflammation may also serve as a proximate mediator to functional decline, ${ }^{18}$ and to frailty development in aging. ${ }^{19}$

\section{NON-AIDS COMPLICATIONS IN AGING HIV-INFECTED PATIENTS}

One of the important studies of the ART era was the Strategies for Management of Antiretroviral Therapy (SMART). ${ }^{20}$ It was designed to compare two treatment strategies: one which was viral suppressive and continuous regardless of $\mathrm{CD} 4$ count; the other with treatment interruptions according to $\mathrm{CD} 4$ levels. After a mean follow-up period of 16 months, the study review board recommended to stop enrollment to the trial because of a safety risk in the treatment interruption group. The statistical analysis showed that patients in the interruption group had an increased risk of mortality, both from opportunistic infections and from cardiovascular, renal, or hepatic disease. This study demonstrated the health effects of HIV beyond AIDS-defining illnesses.

\section{METABOLIC CHANGES AND CARDIOVASCULAR DISEASE}

The HIV-positive population experiences both external and internal metabolic changes. Abnormal fat distribution, also known as lipodystrophy, occurs in both treated ${ }^{21}$ and untreated ${ }^{22}$ HIV-positive patients. It includes two different syndromes: lipoatrophy, or subcutaneous fat loss of face, extremities, and buttocks; and lipohypertrophy, or central fat deposition, manifested as intra-abdominal (visceral) fat, buffalo hump, or breast enlargement. The risk factors for the two abnormal fat distribution 
syndromes are different. According to the Fat Redistribution and Metabolic Change in HIV Infection (FRAM) study, lipoatrophy can be found in almost $40 \%$ of HIV-positive men ${ }^{23}$ and $30 \%$ of HIVpositive women. ${ }^{24}$ Patients at higher risk to develop lipoatrophy are the ones with lower BMI (body mass index), higher nadir HIV load, and use of ART, especially stavudine, zidovudine, and earlier protease inhibitors (PIs). ${ }^{25}$ Lipohypertrophy is more common in HIV-positive women than HIV-positive men and in individuals with greater body fat levels to begin with. ${ }^{26}$

HIV-positive patients with abnormal fat distribution have significantly increased prevalence of dyslipidemia and impaired glucose homeostasis in comparison with HIV-negative controls matched for age and BMI. ${ }^{27}$ The dyslipidemia associated with HIV infection itself includes elevated triglyceride levels and decreased high-density lipoprotein cholesterol (HDL-C) levels. ART is also a major contributor to dyslipidemia, mainly a more profound elevation of triglycerides with ritonavir-based PI regimens. ${ }^{28}$ Likewise, both decreased subcutaneous leg fat and increased visceral fat are strongly associated with decreased insulin sensitivity in this population. ${ }^{29}$ In addition, ART may have an effect on insulin sensitivity, mainly the PIs. One of the mechanisms by which PIs induce insulin resistance is through blocking the transport of glucose by the insulin-sensitive glucose transporter GLUT4. ${ }^{30} \mathrm{~A}$ prospective 10-year follow-up of 1,046 ART-treated HIV-positive patients demonstrated an increased incidence of diabetes mellitus in comparison to the general population, and the risk factors were older age, adiposity, and short exposure to the PI indinavir and the nucleoside reverse transcriptase inhibitors (NRTIs) stavudine and didanosine, ${ }^{31}$ which are mostly not used today in the developed world.

The combination of metabolic and immunologic changes are the base of cardiovascular disease (CVD) in HIV-positive patients. ${ }^{2}$ In addition to the established risk factors for coronary heart disease (CHD) in the general population, which have been shown to be increased in the HIV-positive population, 33 there is additional risk that might be explained in part by both antiretroviral medications and novel CHD risk factors including inflammation and immune dysfunction. The effect of ART was assessed in the Data Collection on Adverse Events of Anti-HIV Drugs (DAD) study, which demonstrated an association between duration of exposure to combination ART and the risk of myocardial infarction, specifically with exposure to PIs. ${ }^{34}$ In contrast, a large study from the Veteran Affairs (VA) system showed no connection between any ART class and CHD or cerebrovascular event outcomes.

Several surrogate indices of CVD have been tested in HIV-positive patients. A recent study demonstrated an association between immune activation markers and carotid artery plaque in patients virologically suppressed on ART, and another study demonstrated elevated carotid intima-media thickness in all HIV groups versus controls, including elite-controllers (HIV-infected patients who maintain an undetectable HIV RNA by standard assay in the absence of ART). 35 The same trend was demonstrated with increased prevalence of subclinical coronary atherosclerosis detected by coronary computed tomography angiography in HIV-infected men in comparison with controls. ${ }^{36}$

The actual increased risk for CHD and acute myocardial infarction in HIV-positive patients was shown in several studies, which found significantly increased risk ratios up to 1.94 (95\% CI 1.58-2.37).37

\section{Renal Complications}

The pathogenesis of renal disease in HIV-positive individuals is diverse. It includes: 1) HIV-associated nephropathy (HIVAN), a form of focal segmental glomerulosclerosis that is accompanied by tubuleinterstitial inflammation, and clinically manifests as rapidly progressive renal failure with nephritic range proteinuria. 2) HIV immune complex kidney disease (HIVICK), a collective term that includes IgA nephropathy, membranoproliferative glomerulonephritis, membranous nephropathy, and a lupus-like glomerulonephritis that is serologically negative.$^{8}$ 3) Hypertensive and atherosclerotic renal disease. 4) ART side-effects, mainly tenofovirinduced renal tubular injury 39 and indinavir/ atazanavir-induced crystaluria and renal calculi formation. ${ }^{40}$ The first two pathologies are more common in untreated patients, the last two in treated. It has been shown that chronic kidney disease and proteinuria are associated with increased risk of mortality in HIV-positive patients. ${ }^{41}$

\section{Bone Mineral Density and Osteoporosis}

Several population-based studies in the United States showed increased prevalence of osteoporotic fractures in HIV-infected men and women compared with HIV-uninfected individuals. ${ }^{2}$ The etiology of low bone mineral density (BMD) in HIV- 
positive patients is multifactorial. It includes both traditional, non-HIV-related risk factors such as smoking, alcohol and opiate use, low body weight, and vitamin D deficiency; and also HIV-related factors such as direct viral and inflammatory effects on bone resorption 43,44 and the effects of ART, especially tenofovir. 45 Multiple studies have shown a 2\%-6\% BMD loss after 48-96 weeks of therapy, regardless of the type of ART initiated. ${ }^{46}$ Several longitudinal studies have shown that, with continued ART use, BMD stabilizes over time. ${ }^{47,48}$

\section{Neurocognitive Changes}

HIV-associated neurocognitive disorder (HAND) is divided into three levels of impairment: asymptomatic neurocognitive impairment, mild neurocognitive disorders, and HIV-associated dementia (HAD). The introduction of ART has reduced significantly the rate of $\mathrm{HAD}$, but unfortunately the effect on less severe forms of impairment is not as impressive. Studies of HAND in treated patients have documented high persisting rates of mild-tomoderate neurocognitive impairment despite effective suppressing antiretroviral treatment, 49 especially in individuals with a history of low nadir CD4s.50

\section{Frailty Syndrome in HIV-positive Older Adults}

Frailty is defined as a syndrome of decreased physiological reserve, which increases vulnerability to negative outcomes such as loss of independence, nursing home admission, morbidity, and mortality. ${ }^{51}$ Recent studies demonstrated that HIV-positive individuals are at an increased risk of frailty and that some individuals with HIV manifest frailty characteristics at a much younger age than frail individuals without HIV ${ }^{6}$ In the pre-ART era frailty in HIV was connected to the AIDS-wasting syndrome, with advanced immunosuppression and very high viral loads. In contrast, the current risk factors for frailty in the HIV-positive population is high fat mass, particularly trunkal fat, and high BMI.52

\section{CONCLUSION}

Accelerated aging of the immune system together with earlier appearance of aging co-morbidities (Figure ) in HIV patients point to a potential major contribution of immune system dysfunction to the accelerated aging in HIV-infected patients. This may once again highlight the role of normal immune function as a critical factor in the fight against HIV

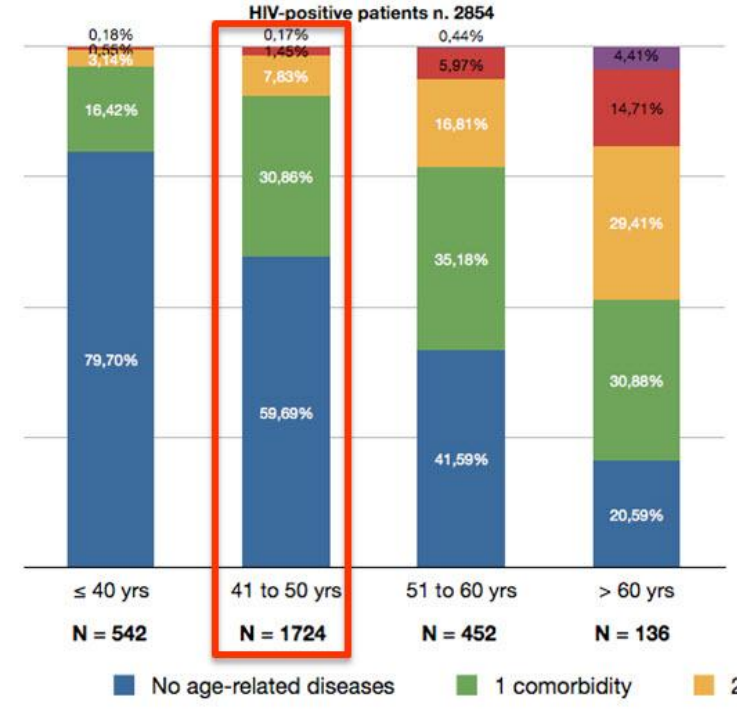

\section{Pp $3.9 \%$ \\ $20.0 \%$ \\ $46.9 \%$}

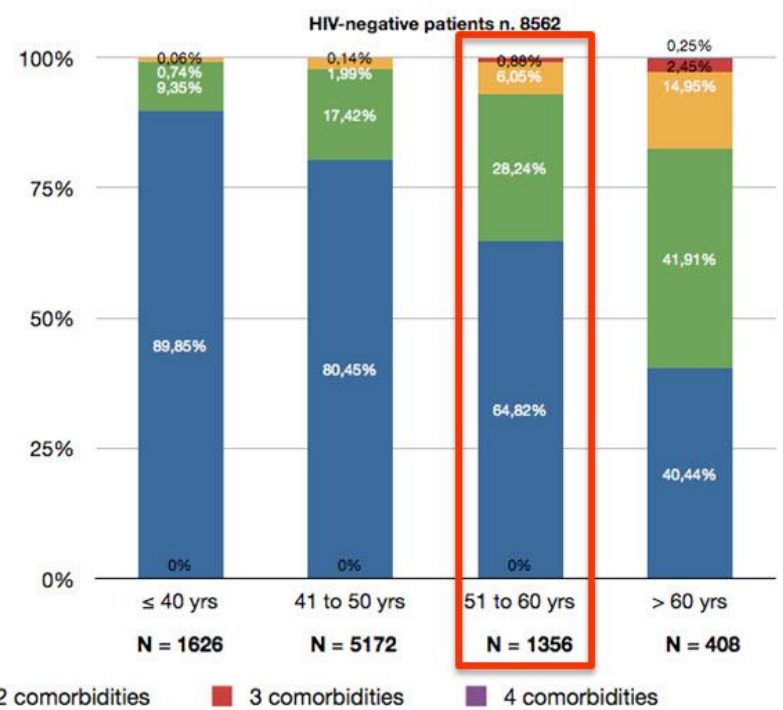

2 comorbidities

3 comorbidities

4 comorbidities

$\begin{array}{llll}\text { Pp } & 0.5 \% & 1.9 \% & 6.6 \%\end{array}$

$18.7 \%$

Figure 3. Poly-patology (Pp) prevalence of age-related non-AIDS conditions in HIV-positive versus HIV-negative populations, 2002-2008.

Modified from Guaraldi $G$ et al., ${ }^{53}$ with permission. 
which, if successful, may both suppress HIV and also attenuate the process of accelerated aging. Successful cART is critical to the recovery of the immune system in HIV-infected individuals. Early initiation of antiretroviral therapy once HIV diagnosis has been established, which will probably keep the normal function of the immune system, may help in alleviating at least some of the morbid conditions related to accelerated aging. We will be able to verify this hypothesis once the results of the on-going international large study, testing the right time to start cART (START study), come out.54

\section{REFERENCES}

1. Mildvan D, Mathur U, Enlow RW, et al. Opportunistic infections and immune deficiency in homosexual men. Ann Intern Med 1982;96(6 Pt 1):700-4.

2. UNAIDS Data Tables $\mid$ 2011. Published by the Joint United Nations Programme on HIV/AIDS (UNAIDS). Available at: http://tinyurl.com/8qinw63. Accessed October 2012.

3. Lohse N, Eg Hansen A-B, Pedersen G, et al. Survival of persons with and without HIF infection in Denmark, 1995-2005. Ann Intern Med 2007;146(2): 87-95.

4. Growing Older with the Epidemic: HIV and Aging. New York, NY: Gay Men's Health Crisis, Inc. 2010.

5. Effros RB, Fletcher CV, Gebo K, et al. Aging and infectious diseases: workshop on HIV infection and aging: what is known and future research directions. Clin Infect Dis 2008;47:542-53.

6. Guaraldi G, Orlando G, Zona S, et al. Premature agerelated comorbidities among HIV-infected persons compared with the general population. Clin Infect Dis 2011;53:1120-6. Full Text

7. Bhatia R, Ryscavage P, Taiwo B. Accelerated aging and human immunodeficiency virus infection: emerging challenges of growing older in the era of successful antiretroviral therapy. J NeuroVirology 2012;18:247-55. Full Text

8. Desquilbet L, Jacobson LP, Fried LP, et al. HIV-1 infection is associated with an earlier occurrence of a phenotype related to frailty. J Gerontol A Biol Sci Med Sci 2007;62:1279-86. Full Text

9. Kalayjian RC, Landay A, Pollard RB, et al. Agerelated immune dysfunction in health and in human immunodeficiency virus (HIV) disease: association of age and HIV infection with naive CD8+ cell depletion, reduced expression of CD28 on CD8+ cells, and reduced thymic volumes. J Infect Dis 2003;187: 1924-33. Full Text
10. Tsaknaridis L, Spencer L, Culbertson $\mathrm{N}$, et al. Functional assay for human CD4+CD25+ Treg cells reveals an age-dependent loss of suppressive activity. J Neurosci Res 2003;74:296-308. Full Text

11. Kogut I, Scholz JL, Cancro MP, Cambier JC. B cell maintenance and function in aging. Semin Immunol 2012; May 3 [Epub ahead of print]. Full Text

12. Rickabaugh TM, Kilpatrick RD, Hultin LE, et al. The dual impact of HIV- 1 infection and aging on naïve CD4 T-cells: additive and distinct patterns of impairment. PLoS One 2011;6:e16459. Full Text

13. Hearpsa AC, Maisaa A, Chenga W-J, et al. HIV infection induces age-related changes to monocytes and innate immune activation in young men that persist despite combination antiretroviral therapy. AIDS 2012;26:843-53. Full Text

14. Bestilny LJ, Gill MJ, Mody CH, Riabowol KT. Accelerated replicative senescence of the peripheral immune system induced by HIV infection. AIDS 2000;14:771-80. Full Text

15. Van Baarle D, Tsegaye A, Miedema F, Akbar A. Significance of senescence for virus-specific memory $\mathrm{T}$ cell responses: rapid ageing during chronic stimulation of the immune system. Immunol Lett 2005;97:19-29. Full Text

16. Neuhaus J, Jacobs DR Jr, Baker JV, et al. Markers of inflammation, coagulation, and renal function are elevated in adults with HIV infection. $J$ Infect Dis 2010;201:1788-95. Full Text

17. Hunt PW, Brenchley J, Sinclair E, et al. Relationship between $\mathrm{T}$ cell activation and $\mathrm{CD} 4+\mathrm{T}$ cell count in HIV-seropositive individuals with undetectable plasma HIV RNA levels in the absence of therapy. $\mathrm{J}$ Infect Dis 2008;197:126-33. Full Text

18. Corsonello A, Garasto S, Abbatecola AM, et al. Targeting inflammation to slow or delay functional decline: where are we? Biogerontology 2010;11:60314. Full Text

19. Bortz WM. A conceptual framework of frailty: a review. J Gerontol A Biol Sci Med Sci 2002;57: M283-8. Full Text

20. Strategies for Management of Antiretroviral Therapy (SMART) Study Group; El-Sadr WM, Lundgren JD, Neaton JD, et al. CD4+ count-guided interruption of antiretroviral treatment. N Engl J Med 2006;355: 2283-96. Full Text

21. Carr A, Samaras K, Burton S, et al. A syndrome of peripheral lipodystrophy, hyperlipidaemia and insulin resistance in patients receiving HIV protease inhibitors. AIDS 1998;12:F51-8. 
22. Madge S, Kinloch-de-Loes S, Mercey D, Johnson MA, Weller IV. Lipodystrophy in patients naive to HIV protease inhibitors. AIDS 1999;13:735-7.

23. Bacchetti P, Gripshover B, Grunfeld C, et al. Study of Fat Redistribution and Metabolic Change in HIV Infection (FRAM). Fat distribution in men with HIV infection. J Acquir Immune Defic Syndr 2005;40: 121-31.

24. Study of Fat Redistribution and Metabolic Change in HIV Infection (FRAM). Fat distribution in women with HIV infection. J Acquir Immune Defic Syndr 2006;42:562-71.

25. Shlay JC, Sharma S, Peng G, Gibert CL, Grunfeld C. The effect of individual antiretroviral drugs on body composition in HIV infected persons initiating highly active antiretroviral therapy. J Acquir Immune Defic Syndr 2009;51:298-304. Full Text

26. Jacobson DL, Knox T, Spiegelman D, Skinner S, Gorbach S, Wanke C. Prevalence of, evolution of, and risk factors for fat atrophy and fat deposition in a cohort of HIV-infected men and women. Clin Infect Dis 2005;40:1837-45. Full Text

27. Hadigan C, Meigs JB, Corcoran C, et al. Metabolic abnormalities and cardiovascular disease risk factors in adults with human immunodeficiency virus infection and lipodystrophy. Clin Infect Dis 2001;32: 130-9. Full Text

28. Pao V, Lee GA, Grunfeld C. HIV therapy, metabolic syndrome, and cardiovascular risk. Curr Atheroscler Rep 2008;10:61-70. Full Text

29. Grunfeld C, Rimland D, Gibert CL, et al. Association of upper trunk and visceral adipose tissue volume with insulin resistance in control and HIV-infected subjects in the FRAM study. J Acquir Immune Defic Syndr 2007;46:283-90. Full Text

30. Murata H, Hruz PW, Mueckler M. The mechanism of insulin resistance caused by HIV protease inhibitor therapy. J Biol Chem 2000;275:20251-4. Full Text

31. Capeau J, Bouteloup V, Katlama C, et al.; ANRS CO8 APROCO-COPILOTE Cohort Study Group. Ten-year diabetes incidence in $1046 \mathrm{HIV}$-infected patients started on a combination antiretroviral treatment. AIDS 2012;26:303-14. Full Text

32. Hsue PY, Deeks SG, Hunt PW. Immunologic basis of cardiovascular disease in HIV-infected adults. J Infect Dis 2012;205:S375-82. Full Text

33. Triant VA, Lee $\mathrm{H}$, Hadigan C, Grinspoon SK. Increased acute myocardial infarction rates and cardiovascular risk factors among patients with human immunodeficiency virus disease. J Clin Endocrinol Metab 2007;92:2506-12. Full Text
34. DAD Study Group; Friis-Møller N, Reiss P, Sabin CA, et al. Class of antiretroviral drugs and the risk of myocardial infarction. N Engl J Med 2007;356:172335. Full Text

35. Hsue PY, Hunt PW, Schnell A, et al. Role of viral replication, antiretroviral therapy, and immunodeficiency in HIV-associated atherosclerosis. AIDS 2009;23:1059-67. Full Text

36. Lo J, Abbara S, Shturman L, et al. Increased prevalence of subclinical coronary atherosclerosis detected by coronary computed tomography angiography in HIV-infected men. AIDS 2010;24:243-53. Full Text

37. Freiberg M, McGinnis K, Butt A, et al. HIV is associated with clinically confirmed myocardial infarction after adjustment for smoking and other risk factors. CROI; 2011: Poster No. 809.

38. Bruggeman LA, Bark C, Kalayjian RC. HIV and the kidney. Curr Infect Dis Rep 2009;11:479-85. Full Text

39. Labarga P, Barreiro P, Martin-Carbonero L, et al. Kidney tubular abnormalities in the absence of impaired glomerular function in HIV patients treated with tenofovir. AIDS 2009;23:689-96. Full Text

40. Mocroft A, Kirk O, Reiss P, et al.; for the EuroSIDA Study Group. Estimated glomerular filtration rate, chronic kidney disease and antiretroviral drug use in HIV-positive patients. AIDS 2010;24:1667-78. Full Text

41. Choi A, Scherzer R, Bacchetti P, et al. Cystatin C, albuminuria, and 5-year all-cause mortality in HIV infected persons. Am J Kidney Dis 2010;56:872-82. Full Text

42. Triant VA, Brown TT, Lee H, Grinspoon SK. Fracture prevalence among human immunodeficiency virus (HIV)-infected versus non-HIV-infected patients in a large U.S. healthcare system. J Clin Endocrinol Metab 2008;93:3499-504. Full Text

43. Fakruddin JM, Laurence J. HIV envelope gp12Omediated regulation of osteoclastogenesis via receptor activator of nuclear factor kappa $\mathrm{B}$ ligand (RANKL) secretion and its modulation by certain HIV protease inhibitors through interferon-gamma/ RANKL cross-talk. J Biol Chem 2003;278:48251-8. Full Text

44. Lam J, Takeshita S, Barker JE, Kanagawa O, Ross FP, Teitelbaum SL. TNF-alpha induces osteoclastogenesis by direct stimulation of macrophages exposed to permissive levels of RANK ligand. J Clin Invest 2000;106:1481-8. Full Text

45. Stellbrink HJ, Orkin C, Arribas JR, et al. Comparison of changes in bone density and turnover with 
abacavir-lamivudine versus tenofovir-emtricitabine in HIV-infected adults: 48-week results from the ASSERT study. Clin Infect Dis 2010;51:963-72. Full $\underline{\text { Text }}$

46. Brown TT, McComsey GA, King MS, Qaqish RB, Bernstein BM, da Silva BA. Loss of bone mineral density after antiretroviral therapy initiation, independent of antiretroviral regimen. $\mathrm{J}$ Acquir Immune Defic Syndr 2009;51:554-61. Full Text

47. Bolland MJ, Grey AB, Horne AM, et al. Bone mineral density remains stable in HAART-treated HIVinfected men over 2 years. Clin Endocrinol (Oxf) 2007;67:270-5. Full Text

48. Dolan SE, Kanter JR, Grinspoon S. Longitudinal analysis of bone density in human immunodeficiency virus-infected women. J Clin Endocrinol Metab 2006;91:2938-45. Full Text

49. Simioni S, Cavassini M, Annoni JM, et al. Cognitive dysfunction in HIV patients despite long-standing suppression of viremia. AIDS 2010;24:1243-50.

50. Heaton RK, Franklin DR, Ellis RJ, et al.; for the CHARTER and HNRC Groups. HIV-associated neurocognitive disorders before and during the era of combination antiretroviral therapy: differences in rates, nature, and predictors. J Neurovirol 2011;17:316. Full Text

51. Ahmed N, Mandel R, Fain MJ. Frailty: an emerging geriatric syndrome. Am J Med 2007;120:748-53. Full Text

52. Shah K, Hilton TN, Myers L, Pinto JF, Luque AE, Hall WJ. A new frailty syndrome: central obesity and frailty in older adults with the human immunodeficiency virus. $\mathrm{J}$ Am Geriatr Soc 2012;60:545-9. Full Text

53. Guaraldi G, Roverato A, Orlando G, et al. Prevalence of poly-pathology is more common in HIV infected patients than in HIV negative controls in any age strata. CROI 2010, Poster 727 (O-209).

54. Babiker AG, Emery S, Fätkenheuer G, et al.; for the INSIGHT START Study Group. Considerations in the rationale, design and methods of the Strategic Timing of AntiRetroviral Treatment (START) study. Clin Trials 2012; April 30 [Epub ahead of print]. Full Text 